\title{
Efficacy and Safety of Moxifloxacin-Enhanced versus Standard Sequential Eradication Therapy for Treating Helicobacter pylori Infection among Tobacco Smokers
}

\author{
Hasanain $\mathrm{AF}^{* 1}$, El-Masry $\mathrm{MA}^{2}$, Soliman $\mathrm{AM}^{3}$, Abd-ellatief $\mathrm{RB}^{4}$, and El-Sers $\mathrm{DA}^{5}$ \\ ${ }^{1}$ Department of Tropical Medicine and Gastroenterology, Faculty of Medicine, Assiut University, Assiut, Egypt \\ ${ }^{2}$ Department of Internal Medicine (Gastroenterology Unit), Faculty of Medicine, Assiut University, Assiut, Egypt \\ ${ }^{3}$ Department of General Surgery (Gastrointestinal and Hepatobiliary Surgery Unit), Faculty of Medicine, Assiut \\ University, Assiut, Egypt \\ ${ }^{4}$ Department of Pharmacology, Faculty of Medicine, Assiut University, Assiut, Egypt \\ ${ }^{5}$ Department of Pathology, Faculty of Medicine, Assiut University, Assiut, Egypt
}

*Corresponding author: Hasanain AF, Department of Tropical Medicine and Gastroenterology, Assiut University, Egypt, Tel: 002-01020288660, E-mail: af.hasanain@outlook.com, a.farooq.hasanain@gmail.com

Citation: Hasanain AF, El-Masry MA, Soliman AM, Abd-ellatief RB, El-Sers DA (2018) Efficacy and Safety of Moxifloxacin-Enhanced versus Standard Sequential Eradication Therapy for Treating Helicobacter pylori Infection among Tobacco Smokers. J Antibio Res 2(1): 101

Received Date: February 16, 2018 Accepted Date: March 14, 2018 Published Date: March 15, 2018

\begin{abstract}
Background: The therapeutic failure rate of eradication therapy for Helicobacter pylori ( $\mathrm{Hp}$ ) is increasing due to bacterial resistance; tobacco smoking can increase such a rate even more. We explored the role of adding moxifloxacin to the standard sequential eradication therapy for $\mathrm{Hp}$ in decreasing the therapeutic failure rate among the tobacco smokers.

Methods: Our study included 193 patients with Hp-associated peptic ulcer disease, consecutively. The patients were randomly allocated to two groups. Group-A included 96 patients who received the standard sequential therapy for eradication of Hp infection (omeprazole $20 \mathrm{mg}$, bid and amoxicillin $1 \mathrm{gm}$ bid, for 5 days, followed by omeprazole, clarithromycin $500 \mathrm{mg}$ bid, and tinidazole $500 \mathrm{mg}$ bid, for 5 days), while group-B included 97 patients who received the moxifloxacin-enhanced sequential therapy (just as the standard sequential therapy with adding moxifloxacin $500 \mathrm{mg}$ qd during the first 5 days). Diagnosis of Hp infection and determination of its eradication were based on the results of rapid urease test and histopathologic examination of mucosal specimens from stomach and duodenum obtained by endoscopic biopsy.

Results: The study population groups were matching; the mean age of the study patients was $36.4 \pm 11.8$ for group-A, while it was $41.2 \pm 14.3$ for group-B. The eradication rate of $\mathrm{Hp}$ was significantly higher among patients of group-B compared to those of group-A (81.4\% (95\% CI 72.6-88.9\%) vs 57.3\% (48.5-65.6\%), p 0.038). Although the adverse effects were more frequent among patients of group-B compared to those of group-A (18.6\% vs $12.5 \%$, p 0.083), this was not statistically significant except for diarrhea.

Conclusions: The moxifloxacin-enhanced, sequential therapy for eradication of Hp infection is more efficacious than the standard, sequential therapy. The higher efficacy was not on the expense of safety; the enhanced, sequential therapy is not significantly less safe than the standard one.
\end{abstract}

Keywords: Sequential therapy; Helicobacter pylori; Tobacco smokers; Moxifloxacin; Quinolones

List of abbreviations: Hp: Helicobacter pylori; PPI: Proton pump inhibitor;

\section{Introduction}

Almost half of the global population is infected with Helicobacter pylori (Hp) [1]. The infection is a well-recognized cause of gastritis, gastroduodenal ulcers, gastric mucosa-associated lymphoid tissue lymphoma, and gastric cancer [2]. The effect of infection is not limited to the stomach; it leads to several extra-gastric disorders [3]. No single combination therapy for eradication of $\mathrm{Hp}$ has achieved $100 \%$ cure rate yet. The recommended regimen for eradication of $\mathrm{Hp}$ is the standard concomitant therapy, which consists of a proton-pump inhibitor (PPI) and two antibiotics (amoxicillin plus clarithromycin) for 14 days [4,5]. The efficacy of this regimen decreased to unacceptable levels due to the development of bacterial resistance [6]. In addition, tobacco 
smoking decreases the therapeutic response of Hp infection to the standard concomitant therapy [7].

As an effort to increase Hp eradication rates, the sequential therapy was adopted. It consists of a dual therapy (PPI and amoxicillin, for five days), followed by a triple therapy (PPI, clarithromycin, and tinidazole/metronidazole, for more five days) [8]. The standard sequential therapy achieved satisfactory rates of eradication; however, it is not recommended if the combined clarithromycin and metronidazole resistance is over 5\% [9]. Levofloxacin-based sequential therapy (replacing clarithromycin with levofloxacin) was preferred by many physicians, in a trial to overcome the bacterial resistance to the standard clarithromycin-based sequential therapy [10]. Unfortunately, the modified levofloxacin-based sequential therapy achieved unsatisfactory eradication rate (79\%) compared to the standard clarithromycin-based sequential therapy (90\%) [11]. However, two weeks of moxifloxacin-based concomitant therapy (PPI, and amoxicillin plus moxifloxacin) achieved $80 \%$ eradication rate of Hp infection among difficult-to-treat patients, who failed non-bismuth quadruple therapy [12]. In addition, five days of levofloxacin-containing quadruple concomitant therapy were as efficacious and safe as 10 days of levofloxacin-containing sequential therapy for eradicating $H$ pylori infection [13].

Our research hypothesis is based on the increasing therapeutic failure rate of eradication therapy for Hp due to the development of bacterial resistance; this rate can even be higher with tobacco smoking. We explored the role of adding moxifloxacin to the standard sequential eradication therapy for $\mathrm{Hp}$ in decreasing the rate of therapeutic failure among the tobacco smokers.

\section{Patients and Methods}

\section{Study design}

A randomized controlled clinical trial was conducted.

\section{Study location}

The study population was recruited from the patients attending the Gastrointestinal Endoscopy Unit.

\section{Study duration}

The study population was recruited during the period from April 2015 to August 2017.

\section{Inclusion criteria}

Our study included 200 patients with Hp-associated peptic ulcer disease, consecutively. Only current tobacco smokers were included. The Hp infection was diagnosed based on positive results of both rapid urease test and histopathologic examination (with modified Giemsa stain) of mucosal specimens from stomach and duodenum obtained by endoscopic biopsy.

\section{Exclusion criteria}

Pregnant patients, patients less than 18 years old, and those using nonsteroidal anti-inflammatory drugs, PPI, antibiotics, corticosteroids, or alcohol during the previous eight weeks before enrollment were excluded from the study. Also, patients with history of gastroduodenal surgery and neoplasms, and those with diabetes mellitus, liver cirrhosis, and renal failure were not included.

\section{Methods}

For all the enrolled patients, clinical evaluation (medical history and physical examination), abdominal ultrasonography, esophagogastroduodenoscopy (with biopsy of gastric and duodenal mucosa, and rapid urease test of the mucosal specimens), and laboratory evaluation were provided. Laboratory evaluation included histopathological examination of mucosal specimens from stomach and duodenum obtained with endoscopic biopsy, using modified Giemsa stain. In addition, estimation of fasting serum level of glucose, serum level of creatinine, and liver chemistry profile were performed. Esophagogastroduodenoscopy was repeated eight weeks after omeprazole therapy. Eradication of Hp infection was determined based on negative results of both rapid urease test and histopathological examination (with modified Giemsa stain) of endoscopically-obtained mucosal specimens from stomach and duodenum. Healing of peptic ulcers was also determined by endoscopy.

The patients were randomly allocated, by a computer-generated stratified random assignments list (block randomization), to two groups. Group-A (considered as a positive control group) included 100 patients who received the standard sequential therapy for eradication of $\mathrm{Hp}$ infection, while group-B included another 100 patients who received the moxifloxacin-enhanced sequential therapy. The standard sequential therapy consisted of five days of dual therapy (omeprazole 20 mg capsules twice daily before breakfast and dinner by 30 minutes and amoxicillin $1000 \mathrm{mg}$ tablets twice daily after breakfast and dinner) followed by triple therapy for five more days (omeprazole $20 \mathrm{mg}$ capsules twice daily before breakfast and dinner by 30 minutes, clarithromycin $500 \mathrm{mg}$ tablets twice daily after breakfast and dinner, and tinidazole $500 \mathrm{mg}$ tablets twice daily after breakfast and dinner). The moxifloxacin-enhanced sequential therapy consisted of five days of triple therapy (omeprazole $20 \mathrm{mg}$ capsules twice daily before breakfast and dinner by 30 minutes, amoxicillin $1000 \mathrm{mg}$ tablets twice daily after breakfast and dinner, and moxifloxacin $400 \mathrm{mg}$ tablets once daily after lunch by two hours) followed by triple therapy for five more days (omeprazole 20 mg capsules twice daily 
before breakfast and dinner by 30 minutes, clarithromycin $500 \mathrm{mg}$ tablets twice daily after breakfast and dinner, and tinidazole 500 mg tablets twice daily after breakfast and dinner). Each single drug was provided by only one pharmaceutical company to eliminate the possibility of having difference in the bioavailability related to the production process by different pharmaceutical companies.

\section{Statistical analysis}

Data were analyzed using the Statistical Package for Social Sciences (IBM SPSS Statistics, version 22.0, release 22.0.0.0; IBM Corp) for Microsoft Windows ${ }^{\circledast}$ (64-bit version). Results were expressed as mean \pm standard deviation or frequency (percentage) as appropriate. Student's t-test or Mann-Whitney U test, and Yates' corrected chi-squared test or Fischer's exact test as appropriate were used to compare the variables between the study groups. A p value less than 0.05 was considered statistically significant.

Power analysis of the performed chi-square tests was done using the $\mathrm{G}^{\star}$ power software version 3.1.9.2. A power of $84 \%$ was achieved to detect a medium sized effect. Plotting power against various effect size values showed reasonable power values for medium and large effect sizes. However, for detection of small effect size, the test appeared to be underpowered.

\section{Ethical considerations}

The study was conducted after approval of the Clinical Research Ethical Committee of Assiut Faculty of Medicine and was carried out according to the code of ethics of the World Medical Association (Declaration of Helsinki). All the participants signed a consent certificate after discussing in detail with the investigators the certificate subjects and the study aim. Participants were clearly informed that refusing to participate in the study will not affect having full benefit of the available medical service. Data confidentiality was respected.

\section{Results}

After exclusion of four patients of group-A and three patients of group-B (for not undergoing follow-up esophagogastroduodenoscopy after eight weeks of omeprazole therapy), the study included 193 eligible patients (96 of group-A and 97 of group-B). All the cases of hp infection were detected in gastric mucosa, with only $43 \%$ of them detected simultaneously in duodenal mucosa. The demographic, clinical, and endoscopic characteristics of the study population are shown in Table 1. Both groups were matching; the mean age of the study population was $36.4 \pm 11.8$ for group-A, while it was $41.2 \pm 14.3$ for group-B. Most of the study population was males ( $96 \%$ of patients of group-A and $94 \%$ of those of group-B). The most frequent indication for esophagogastroduodenoscopy among patients of group-A was dyspepsia (51\%), while it was epigastric pain (57\%) among those of group-B. Some patients had more than one indication. Endoscopy revealed duodenal ulcers as the most frequent endoscopic lesion among both groups of the study population (74\% among patients of group-A, and 69\% among those of group-B). Gastric ulcers were less frequent than duodenal ulcers (26\% among patients of group-A, and $34 \%$ among those of group-B). Approximately, one-third of the study population of both groups was treatment-experienced (received eradication therapy of Hp more than eight weeks before enrollment in the study). The only extra-gastroduodenal lesions detected on endoscopy were lower esophagitis and lower esophageal tears. Some patients had more than one lesion. Concomitant gastric and duodenal ulcers existed among 3 patients of group-B.

\begin{tabular}{|c|c|c|c|}
\hline & Group-A $(n=96)$ & Group-B (n-97) & $p$ \\
\hline Age (years) & $36.4 \pm 11.8$ & $41.2 \pm 14.3$ & 0.294 \\
\hline Gender & & & 0.671 \\
\hline Females & $4(4.2)$ & $6(6.2)$ & \\
\hline Males & $92(95.8)$ & $91(93.8)$ & \\
\hline Treatment experienced * & $29(30.2)$ & $35(36.1)$ & 0.506 \\
\hline \multicolumn{4}{|l|}{ Indications for endoscopy } \\
\hline Dyspepsia & $49(51)$ & $42(43.3)$ & \\
\hline Epigastric pain & $47(49)$ & $55(56.7)$ & \\
\hline Heart burn & $12(12.5)$ & $8(8.3)$ & \\
\hline Vomiting & $7(7.3)$ & $11(11.3)$ & \\
\hline Dysphagia & $2(2.1)$ & $1(1)$ & \\
\hline Hematemesis & $9(9.4)$ & $13(13.4)$ & \\
\hline Anemia & $12(12.5)$ & $15(15.5)$ & \\
\hline \multicolumn{4}{|l|}{ Endoscopic findings } \\
\hline Gastric ulcers & $25(26)$ & $33(34)$ & 0.082 \\
\hline Gastric erosions & $36(37.5)$ & $41(42.3)$ & 0.334 \\
\hline Gastritis & $61(63.5)$ & $64(66)$ & 0.607 \\
\hline Duodenal ulcers & $71(74)$ & $67(69.1)$ & 0.496 \\
\hline
\end{tabular}




\begin{tabular}{|c|c|c|c|}
\hline & Group-A (n=96) & Group-B (n-97) & $\boldsymbol{p}$ \\
\hline Duodenal erosions & $42(43.8)$ & $39(40.2)$ & 0.551 \\
\hline Duodenitis & $28(29.2)$ & $30(30.9)$ & 0.174 \\
\hline Lower esophagitis & $11(11.5)$ & $9(9.3)$ & 0.298 \\
\hline Lower esophageal tears & $4(4.2)$ & $3(3.1)$ & 0.163 \\
\hline
\end{tabular}

Hp: Helicobacter pylori; n: number.

Group-A: patients receiving standard, sequential eradication therapy (omeprazole, amoxicillin, clarithromycin, and tinidazole); group-B: patients receiving moxifloxacin-enhanced, sequential eradication therapy (omeprazole, amoxicillin, moxifloxacin, clarithromycin, and tinidazole).

Data are presented as frequency (percentage), except for age which is presented as mean \pm standard deviation.

* Received eradication therapy of Hp more than eight weeks before enrollment in the study.

Table 1: Demographic, clinical, and endoscopic characteristics of the study population with Hp infection

Table 2 shows the therapeutic outcome among the study population after eradication therapy and eight weeks of omeprazole therapy. The therapeutic failure rate of eradication therapy for $\mathrm{Hp}$ infection was significantly lower among patients of group-B compared to those of group-A (18.6\% (95\% CI 13.5-24.7\%) vs 42.7\% (33.2-54.8\%), p 0.038) according to per-protocol analysis. Using intention-to-treat analysis, the results did not differ ( $18 \%$ vs $41 \%, p 0.019)$. Although the rate of healing for gastroduodenal lesions (ulcers, erosions, and inflammation) was higher among patients of group-B compared to those of group-A, this was not statistically significant. However, when considering each endoscopic lesion separately, the rates of healing for duodenal ulcers and duodenal erosion were significantly higher among patients of group-B compared to those of group-A (77.6\% (95\% CI 68.3-89.7\%) vs $47.9 \%$ (95\% CI 41.6-60.1\%), p 0.044; 92.3\% (95\% CI 86.9-98.4\%) vs 57.1\% (95\% CI 45.7-70.2\%), p 0.030), respectively. Figure-1 illustrates the therapeutic outcome of the study population. Among patients with Hp eradication, the healing rate of peptic ulcer disease was $92.7 \%$ among patients of group-A, while it was $93.7 \%$ among those of group-B.

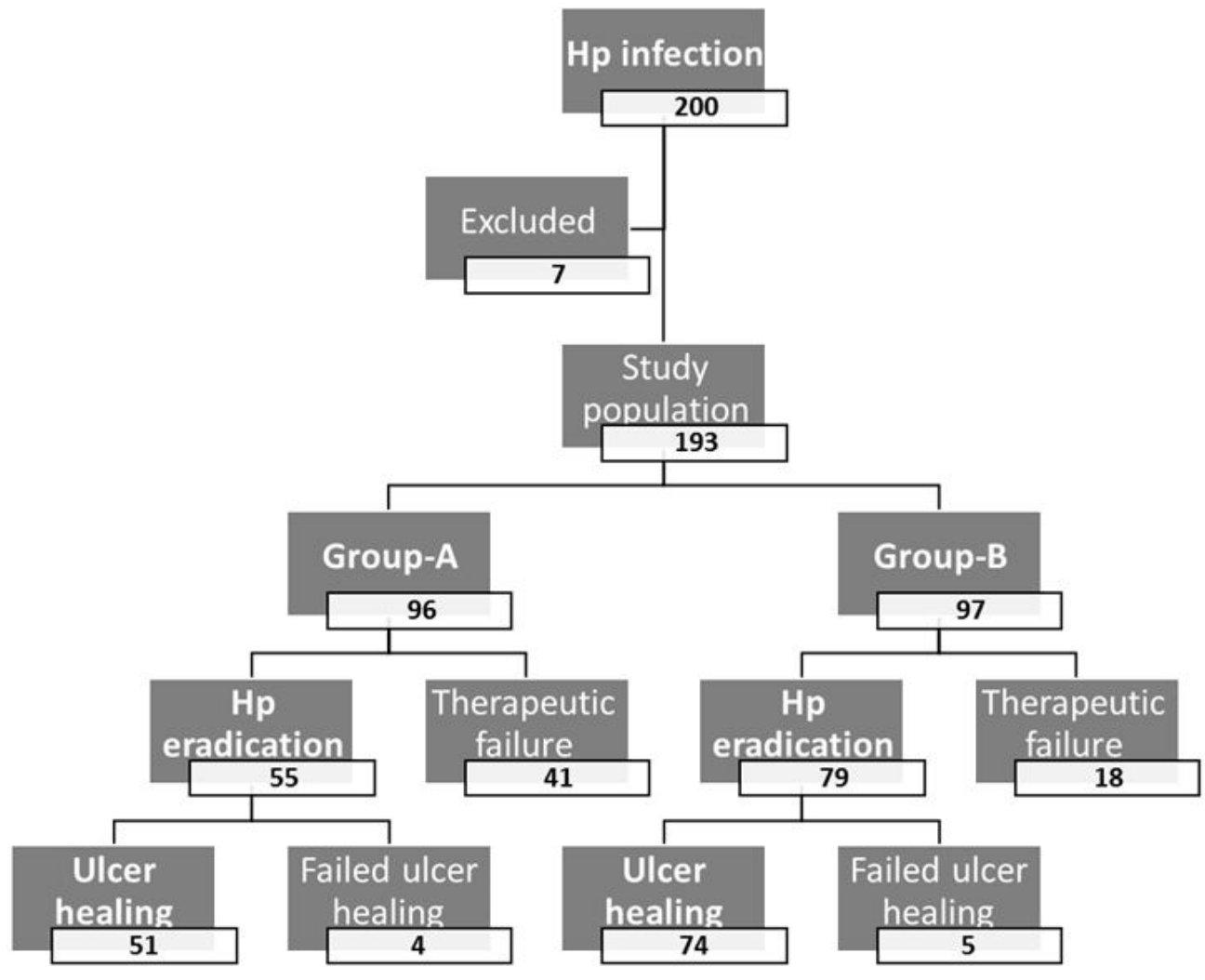

Figure 1: Flow chart of the study population with Hp infection. Hp: Helicobacter pylori

\begin{tabular}{|c|c|c|c|}
\hline & Group-A (n=96) & Group-B (n=97) & $\boldsymbol{p}$ \\
\hline Hp infection eradication $^{* *}$ & $55 / 96(57.3)$ & $79 / 97(81.4 \%)$ & 0.038 \\
\hline Therapeutic failure $^{* *}$ & $41 / 96(42.7)$ & $18 / 97(18.6)$ & $0.021^{*}$ \\
\hline Ulcer healing & $51 / 96(53.1)$ & $74 / 97(76.3)$ & 0.061 \\
\hline Gastric ulcers & $17 / 25(68)$ & $22 / 33(66.7)$ & 0.416 \\
\hline Duodenal ulcers & $34 / 71(47.9)$ & $52 / 67(77.6)$ & $0.044^{*}$ \\
\hline Erosions healing & $49 / 78(62.8)$ & $68 / 80(85)$ & 0.094 \\
\hline Gastric erosions & $25 / 36(69.4)$ & $32 / 41(78.1)$ & 0.153 \\
\hline
\end{tabular}




\begin{tabular}{|c|c|c|c|}
\hline & Group-A (n=96) & Group-B (n=97) & $\boldsymbol{p}$ \\
\hline Duodenal erosions & $24 / 42(57.1)$ & $36 / 39(92.3)$ & $0.030^{*}$ \\
\hline Mucosal inflammation healing & $66 / 89(74.2)$ & $81 / 94(86.2)$ & 0.253 \\
\hline Gastritis & $44 / 61(72.1)$ & $54 / 64(84.4)$ & 0.302 \\
\hline Duodenitis & $22 / 30(73.3)$ & $27 / 30(90)$ & 0.229 \\
\hline Esophageal lesions & $9 / 15(60)$ & $8 / 12(66.7)$ & 0.462 \\
\hline Lower esophagitis & $7 / 11(63.6)$ & $5 / 9(55.6)$ & 0.551 \\
\hline Lower esophageal tears healing & $2 / 4(50)$ & $3 / 3(100)$ & 0.073 \\
\hline
\end{tabular}

n: number; Hp: Helicobacter pylori.

Group-A: patients receiving standard, sequential eradication therapy (omeprazole, amoxicillin, clarithromycin, and tinidazole); group-B: patients receiving moxifloxacin-enhanced, sequential eradication therapy (omeprazole, amoxicillin, moxifloxacin, clarithromycin, and tinidazole).

* Statistically significant.

** According to per-protocol analysis.

Table 2: Therapeutic outcome among the study population

The adverse effects of the eradication therapy for Hp infection were evaluated during the entire period of therapy, and for two weeks after the end of eradication therapy. Some patients had more than one type of adverse effects. Although the adverse effects were more frequent among patients of group-B compared to those of group-A ( $18.6 \%$ vs $12.5 \%, p 0.083)$, this was not statistically significant (Table 3). Using intention-to-treat analysis, the results did not differ ( $18 \%$ vs $12 \%, p 0.077$ ). The Adverse effects did not lead to discontinuation of therapy among the study population. When considering each type of adverse effects separately, only diarrhea was significantly more frequent among patients of group-B compared to those of group-A (8.3\% (95\% CI 5.6-13.8\%) vs $31 \%$ (22.7-39.1\%), p 0.021); however, it was tolerable, and all patients had complete cure after the end of eradication therapy.

\begin{tabular}{|c|c|c|c|}
\hline & Group-A (n=96) & Group-B (n=97) & $\boldsymbol{p}$ \\
\hline Diarrhea & $3(3.1)$ & $8(8.3)$ & $0.042^{*}$ \\
\hline Bloating & $4(4.2)$ & $6(6.2)$ & 0.186 \\
\hline Nausea & $8(8.3)$ & $11(11.3)$ & 0.297 \\
\hline Anorexia & $1(1)$ & $2(2.1)$ & 0.102 \\
\hline Skin rash & $1(1)$ & $1(1)$ & 0.791 \\
\hline Headache & $2(2.1)$ & $3(3.1)$ & 0.313 \\
\hline Myalgia & 0 & $1(1)$ & na \\
\hline Total ** & $12(12.5)$ & $18(18.6)$ & 0.083 \\
\hline
\end{tabular}

Hp: Helicobacter plyori; n: number; na: not applicable.

Group-A: patients receiving standard, sequential eradication therapy (omeprazole, amoxicillin, clarithromycin, and tinidazole); group-B: patients receiving moxifloxacin-enhanced, sequential eradication therapy (omeprazole, amoxicillin, moxifloxacin, clarithromycin, and tinidazole).

* Statistically significant.

** Using per-protocol analysis.

Table 3: Adverse effects of Hp eradication therapy among the study population

\section{Discussion}

Although neither the standard nor the moxifloxacin-enhanced regimens achieved an appropriate eradication rate of Hp infection among our study patients, the moxifloxacin-enhanced sequential therapy yielded a significantly improved therapeutic response compared to that of the standard sequential therapy. To our knowledge, this is the first time to investigate this moxifloxacin-enhanced, sequential therapy for eradication of $\mathrm{Hp}$ infection. The new, moxifloxacin-enhanced sequential eradication therapy provided a higher efficacy than the standard one, without significantly compromising the patient safety. Only diarrhea was significantly more frequent among our study patients using moxifloxacin-enhanced sequential therapy compared to those who used the standard sequential therapy. Prolonged QT interval can occur with the combined use of quinolones with clarithromycin, through concentration-dependent blockade of the rapidly activating delayed rectifier potassium channel, which may lead to dysrhythmia [14]. However, no single case was detected among our study patients. Even the increase in the cost of treatment overweighs the financial burden of the therapeutic failure with subsequent complications of persistent Hp infection, and consequently peptic ulcer disease. The use of bismuth-based rescue therapy instead of moxifloxacin-based therapy was not possible due to unavailability of bismuth in Egypt.

Moxifloxacin was not used for eradication Hp infection for the first time in our study, although it is the first time to be used added 
to the standard sequential therapy and not as a replacement for one of its antimicrobials. Two weeks of moxifloxacin-containing concomitant therapy was an efficacious and safe second-line regimen for eradication of Hp infection after failure of the standard concomitant triple therapy or non-bismuth quadruple (sequential or concomitant) therapy. Moxifloxacin (400 mg once daily) was used combined with amoxicillin ( $1 \mathrm{gm}$ twice daily) and esomeprazole (40 mg twice daily) [15]. Also, Lim, et al, used moxifloxacinbased, concomitant triple therapy after failure of non-bismuth quadruple therapy to eradicate Hp infection. Moxifloxacin (400 $\mathrm{mg}$ once daily) was used combined with rabeprazole (20 mg twice daily) and amoxicillin ( $1 \mathrm{gm}$ twice daily) for two weeks. This regimen achieved an eradication rate of $80.6 \%$, which was considered as a good therapeutic outcome among the difficult-to-treat patients who failed quadruple therapy. Such patients have a higher potential for bacterial resistance to the antimicrobials of the eradication therapy [12]. Our study also targeted difficult-to-treat patients; the prevalence rate of tobacco smoking was low among those patients (10\%) compared to our study population, who were all tobacco smokers. We had the advantage of recruiting a larger sample size (193 vs 98 patients).

Reports regarding the quinolone-based regimens for eradication of Hp infection are conflicting. While it was reported that using levofloxacin-based concomitant therapy for 10 days yielded $87 \%$ eradication rate [10] and using moxifloxacin-based concomitant therapy for two weeks succeeded to eradicate Hp infection among 90\% of the treated patients [16], it was found that levofloxacinbased sequential therapy were less effective compared to the clarithromycin-based one, yielding lower eradication rate (79\% vs 90\%) after replacing clarithromycin with levofloxacin [11]. In areas with $>15 \%$ clarithromycin-resistant Hp strains, levofloxacincontaining sequential therapy is more effective and equally safe compared to a clarithromycin-containing sequential therapy [17].

Clarithromycin is a back-bone of majority of the regimens used for eradication of Hp infection. The interaction between amoxicillin and clarithromycin is synergistic; amoxicillin destroys a transmembrane efflux system of the bacterial wall that allows elimination of clarithromycin in the resistant bacteria, providing higher intracellular levels of clarithromycin to overcome the mechanisms involved in bacterial resistance [16]. The first five days of therapy using amoxicillin act as an inductor for the next five days of clarithromycin therapy, providing the sequential regimen the ability to eradicate a as much as $82 \%$ of clarithromycin-resistant strains [18]. Instead of replacing it with moxifloxacin, amoxicillin was augmented with moxifloxacin trying to increase the efficacy of the sequential eradication therapy for Hp infection. Moxifloxacin can compensate for the decreased availability of amoxicillin, which is destroyed by higher acid production among tobacco smokers [19]. In addition, synergism between moxifloxacin and clarithromycin was observed among the patients treated for post-acupuncture cutaneous infections due to Mycobacterium abscessus [20]. The use of extended-duration moxifloxacin-containing concomitant therapy for two weeks following standard concomitant therapy did not provide higher eradication rate [21] due to increasing resistance rates to moxifloxacin [22]. Consequently, extending the duration of our enhanced sequential therapy was not considered to avoid the development of bacterial resistance to moxifloxacin. The relatively high eradication rate among our difficult-to-treat patients using the moxifloxacin-enhanced sequential therapy (81.4\%) may reflect a less possibility of bacterial resistance to moxifloxacin among the patients in our locality.

The real challenge of our study was treating a study population who are smoking tobacco. Smoking, a major etiologic factor of peptic ulcer disease, is often associated with therapeutic failure of Hp eradication therapy [23,24]. A therapeutic failure rate of $34.8 \%$ for eradicating Hp infection was reported among smokers receiving the standard concomitant therapy [7]. Through decreasing both gastric blood flow and secretion of mucus, smoking leads to reduced delivery of antimicrobials to the gastric mucosa, and consequently decreasing the efficacy of eradication therapy [25]. As smoking provokes acid secretion, the effect of amoxicillin (acid-sensitive) may be decreased, resulting in failure of Hp eradication therapy [19]. Other mechanisms include poor compliance among smokers [26].

The lack of antibiotic susceptibility testing of Hp before the initiation of treatment is considered as a limitation of our study. However, the matching groups of patients are expected to have almost the same antibiotic resistance pattern. Also, data regarding pack-year quantification of smoking exposure were not available. Still, our study has several strengths. The first, it is a randomized comparative clinical trial including a reasonable number of patients. The second, we relied on both rapid urease test and histopathological examination of gastroduodenal mucosal specimens for diagnosis of Hp infection and for determination of its eradication.

\section{Conclusions}

In conclusion, the moxifloxacin-enhanced, sequential therapy for eradication of Hp infection can be more effective than the standard, sequential therapy. The higher efficacy was not on the expense of safety. The enhanced, sequential therapy resulted in higher frequency of tolerable adverse effects, which did not compromise the patient safety and did not lead to discontinuation of therapy. Before recommending the addition of moxifloxacin to the sequential therapy for eradication of Hp infection, more studies with antibiotic susceptibility testing are needed. Also, other studies comparing the efficacy and safety of moxifloxacin-enhanced sequential therapy between tobacco smokers and nonsmokers are recommended.

\section{References}

1. Eusebi LH, Zagari RM, Bazzoli F (2014) Epidemiology of Helicobacter pylori infection. Helicobacter 19: 1-5.

2. McColl KE (2010) Clinical practice. Helicobacter pylori infection. N Engl J Med 362: 1597-604.

3. Franceschi F, Gasbarrini A, Polyzos SA, Kountouras J (2013) Extragastric diseases and Helicobacter pylori. Helicobacter 18: 44-51. 
4. Lam SK, Talley NJ (1998) Report of the 1997 Asia Pacific Consensus Conference on the management of Helicobacter pylori infection. J Gastroenterol Hepatol 13: 1-12.

5. Malfertheiner P, Megraud F, O’Morain CA, Gisbert JP, Kuipers EJ, et al. (2017) Management of Helicobacter pylori infection-the Maastricht V/Florence Consensus Report. Gut 66: 6-30.

6. Graham DY, Fischbach L (2010) Helicobacter pylori treatment in the era of increasing antibiotic resistance. Gut 59: 1143-53.

7. Itskoviz D, Boltin D, Leibovitzh H, Tsadok Perets T, Comaneshter D, et al. (2017) Smoking increases the likelihood of Helicobacter pylori treatment failure. Dig Liver Dis 49: 764-8.

8. Zullo A, Rinaldi V, Winn S, Meddi P, Lionetti R, et al. (2000) A new highly effective short-term therapy schedule for Helicobacter pylori eradication. Aliment Pharmacol Ther 14: 715-8.

9. Megraud F, Coenen S, Versporten A, Kist M, Lopez-Brea M, et al. (2013) Helicobacter pylori resistance to antibiotics in Europe and its relationship to antibiotic consumption. Gut 62: 34-42.

10. Gisbert JP, Morena F (2006) Systematic review and meta-analysis: levofloxacin-based rescue regimens after Helicobacter pylori treatment failure. Aliment Pharmacol Ther 23: 35-44.

11. Branquinho D, Almeida N, Gregório C, Cabral JE, Casela A, et al. (2017) Levofloxacin or Clarithromycin-based quadruple regimens: what is the best alternative as first-line treatment for Helicobacter pylori eradication in a country with high resistance rates for both antibiotics? BMC Gastroenterol 17: 31.

12. Lim JH, Lee DH, Lee ST, Kim N, Park YS, et al. (2015) Moxifloxacin-containing triple therapy after non-bismuth quadruple therapy failure for Helicobacter pylori infection. World J Gastroenterol 21: 13124-31.

13. Romano M, Cuomo A, Gravina AG, Miranda A, Iovene MR, et al. (2010) Empirical levofloxacin-containing versus clarithromycin-containing sequential therapy for Helicobacter pylori eradication: a randomised trial. Gut 59: 1465-70.

14. Anderson ME, Mazur A, Yang T, Roden DM (2001) Potassium current antagonist properties and proarrhythmic consequences of quinolone antibiotics. J Pharmacol Exp Ther 296: 806-10.

15. Gisbert JP, Romano M, Molina-Infante J, Lucendo AJ, Medina E, et al. (2015) Two-week, high-dose proton pump inhibitor, moxifloxacin triple Helicobacter pylori therapy after failure of standard triple or non-bismuth quadruple treatments. Dig Liver Dis 47: 108-13.

16. De Francesco V, Margiotta M, Zullo A, Hassan C, Troiani L, et al. (2006) Clarithromycin-resistant genotypes and eradication of Helicobacter pylori. Ann Intern Med 144: 94-100.

17. Federico A, Nardone G, Gravina AG, Iovene MR, Miranda A, et al. (2012) Efficacy of 5-day levofloxacin-containing concomitant therapy in eradication of Helicobacter pylori infection. Gastroenterology 143: 55-61.

18. Zullo A, De Francesco V, Hassan C, Morini S, Vaira D (2007) The sequential therapy regimen for Helicobacter pylori eradication: a pooled-data analysis. Gut 56: 1353-7.

19. Moayyedi P, Chalmers DM, Axon AT (1997) Patient factors that predict failure of omeprazole, clarithromycin, and tinidazole to eradicate Helicobacter pylori. J Gastroenterol 32: 24-7.

20. Choi WS, Kim MJ, Park DW, Son SW, Yoon YK, et al. (2011) Clarithromycin and amikacin vs. clarithromycin and moxifloxacin for the treatment of postacupuncture cutaneous infections due to Mycobacterium abscessus: a prospective observational study. Clin Microbiol Infect 17: 1084-90.

21. Chuah SK, Tai WC, Hsu PI, Wu DC, Wu KL, et al. (2012) The efficacy of second-line anti-Helicobacter pylori therapy using an extended 14-day levofloxacin/ amoxicillin/proton-pump inhibitor treatment--a pilot study. Helicobacter 17: 374-81.

22. Yoon H, Kim N, Lee BH, Hwang TJ, Lee DH, et al. (2009) Moxifloxacin-containing triple therapy as second-line treatment for Helicobacter pylori infection: effect of treatment duration and antibiotic resistance on the eradication rate. Helicobacter 14: 77-85.

23. Lee JW, Kim N, Kim JM, Nam RH, Chang H, et al. (2013) Prevalence of primary and secondary antimicrobial resistance of Helicobacter pylori in Korea from 2003 through 2012. Helicobacter 18: 206-14.

24. Kim SE, Park MI, Park SJ, Moon W, Choi YJ, et al. (2015) Trends in Helicobacter pylori eradication rates by first-line triple therapy and related factors in eradication therapy. Korean J Intern Med 30: 801-7.

25. Pan KF, Zhang L, Gerhard M, Ma JL, Liu WD, et al. (2016) A large randomised controlled intervention trial to prevent gastric cancer by eradication of Helicobacter pylori in Linqu County, China: baseline results and factors affecting the eradication. Gut 65: 9-18.

26. Suzuki T, Matsuo K, Ito H, Sawaki A, Hirose K, et al. (2006) Smoking increases the treatment failure for Helicobacter pylori eradication. Am J Med 119: 217-24.

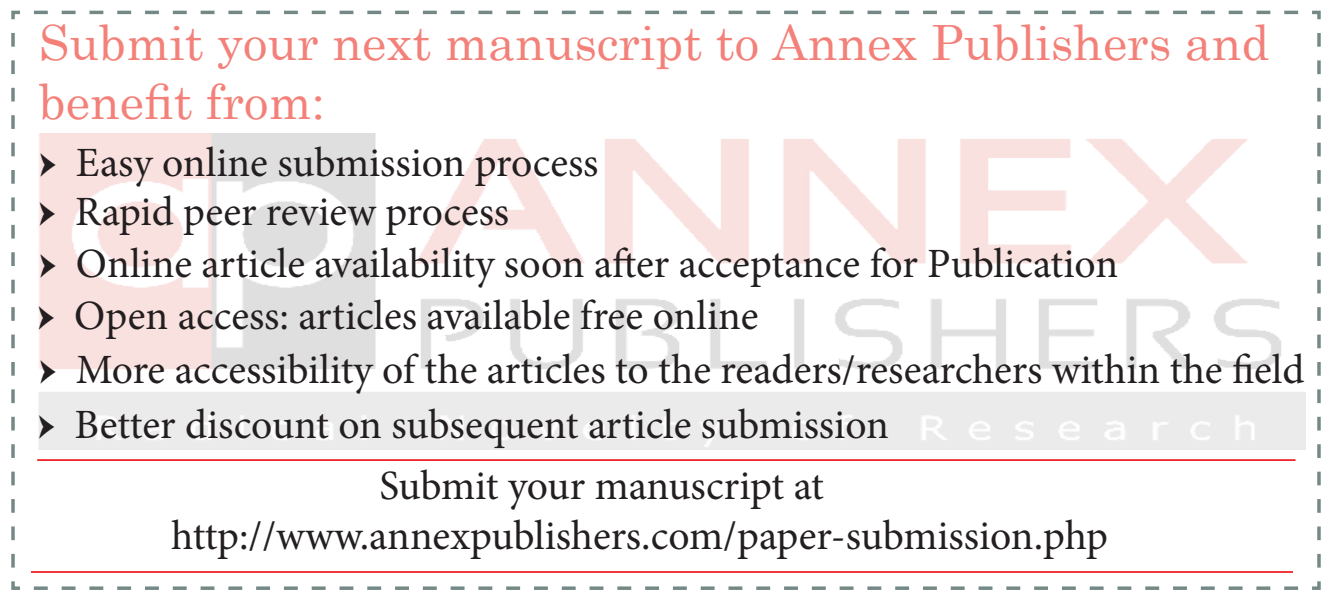

